EGU21-15831, updated on 04 Mar 2021

https://doi.org/10.5194/egusphere-egu21-15831

EGU General Assembly 2021

(c) Author(s) 2021. This work is distributed under

the Creative Commons Attribution 4.0 License.

\title{
Spectral analysis of Apollo Basins on the Moon through spectral units identification
}

Francesca Zambon ${ }^{1}$, Cristian Carli ${ }^{1}$, Francesca Altieri ${ }^{1}$, Jean-Philippe Combe ${ }^{2}$, Carolyn H. van der Bogert ${ }^{3}$, Claudia M. Poehler ${ }^{3}$, Harald Hiesinger ${ }^{3}$, Stephane Le Mouélic ${ }^{4}$, Nicolas Mangold ${ }^{4}$, Gwénaël Caravaca $^{4}$, and Matteo Massironi ${ }^{5,6}$

'INAF - Istituto di Astrofisica e Planetologia Spaziali, via del Fosso del Cavaliere 100, 00133 Roma, Italy

(francesca.zambon@inaf.it)

${ }^{2}$ Bear Fight Institute, 22, Fiddler's Road, P.O. Box 667, Winthrop, WA, 98862, USA

${ }^{3}$ Institut für Planetologie, Westfälische Wilhelms Universität, Wilhelm-Klemm-Str. 10, 48149 Münster, Germany

${ }^{4}$ LPG, UMR CNRS 6112, Université de Nantes, France

${ }^{5}$ University of Padua, Department of Geoscience, Via Gradenigo 6, I-35131 Padua, Italy

${ }^{6}$ INAF - Osservatorio Astronomico di Padova, Vicolo dell'Osservatorio, 5, 35122 Padova, Italy

The spectral analysis of a planetary surface is fundamental for a deeper understanding of the mineralogy and composition. In particular, the determination of spectral units is a reliable method to infer the physical and compositional properties of a surface by processing several spectral parameters simultaneously, instead of the more traditional approach of interpreting each single parameter separately. To define the spectal units, we first compute the most relevant spectral parameters, based on a preliminary detailed analysis of the spectral properties of a surface. This method could be used for different bodies and is described in [1].

For this work, we selected the Apollo Basin area within South Polar Aitken [2,3], the largest and deepest impact basin on the Moon. We analyzed the $\mathrm{M}^{3} /$ Chandrayaan-1 data [4] after performing the most up-to-date calibration, thermal removal and photometric correction [5,6]. Lunar spectra are characterized by two strong pyroxenes absorption bands at 1 and $2 \mu \mathrm{m}$. In this regard, we decided to define the Apollo Basin spectral units by using the two pyroxenes band depths, the reflectance at $540 \mathrm{~nm}$ (standard visible wavelength), and the spectral slope of the $1 \mu \mathrm{m}$ (see [7]). In Apollo Basin, we found 12 different spectral units. Among these units, the most peculiar is the one linked to the basaltic smooth plains within the floor of the crater. This unit is characterized by low reflectance, deep band depths and a strongly positive spectral slopes (more red surfaces). Subsequently, an analysis of absorption band center at 1 and $2 \mu \mathrm{m}$ and a comparison with RELAB synthetic pyroxenes [8] revealed a composition compatible with material dominated by strong pyroxene absorptions, e.g. clinopiroxenes, such as pigeonite or augite, with Low $\mathrm{Ca}$ and $\mathrm{Mg}$, and relatively high Fe (Fs: 34-75; En: 6-23; Wo: 10-27). The rest of the units show a similar mineralogy to the orthopyroxenes, with intermediate amount of Fe and Mg.

This work allows for a detailed understanding of the mineralogy of Apollo Basin, but also lays the groundwork to search for a link between spectral, and morpho-stratigraphic units [9] to reach out 
highly informative geological maps of the Moon. This innovative approach is one of the main goals of the H2020 no. 776276-PLANMAP project [10].

Acknowledgments: This work is funded by the European Union's Horizon 2020 research grant agreement No 776276- PLANMAP.

References: [1] Zambon et al., 2020 LPSC. [2] Ohtake, M. et al., 2014, GRL. [3] Moriarty, D.P. et al., 2018, JGR. [4] Pieters et al., 2009, Current Science. [5] PLANMAP D4.3- Spectral Indices and RGB maps. [6] Besse, S. et al., 2012, Icarus. [7] PLANMAP D4.3- Spectral Indices and RGB maps. [8] http://planetary.brown.edu/relabdocs/synth_pyx/pyroxenes.html. [9] Ivanov, M.A., 2018, JGR. [10] https://www.planmap.eu/. 